\title{
ВЗАИМОСВЯЗЬ АЛЬДОСТЕРОНА И МАРКЕРОВ ФИБРОЗА В ПАТОГЕНЕЗЕ ХРОНИЧЕСКОЙ СЕРДЕЧНОЙ НЕДОСТАТОЧНОСТИ С ПРОМЕЖУТОЧНОЙ ФРАКЦИЕЙ ВЫБРОСА В РАЗНЫХ ВОЗРАСТНЫХ ГРУППАХ
}

\section{RELATIONSHIP OF ALDOSTERONE AND FIBROSIS MARKERS IN THE PATHOGENESIS OF CHRONIC HEART FAILURE BY THE MIDDLE RANGE EJECTION FRACTION IN DIFFERENT AGE GROUPS}

\author{
Е.В. Гостева ${ }^{1}$, Р.Н. Шепель ${ }^{2}$, О.А. Осипова ${ }^{3}$ \\ E.V. Gosteva ${ }^{1}$, R.N. Shepel ${ }^{2}$, O.A. Osipova ${ }^{3}$ \\ ${ }^{1}$ Воронежский государственный медицинский университет имени Н.Н. Бурденко, \\ Россия, 394036, г. Воронеж, Студенческая, 10 \\ ${ }^{2}$ Федеральное государственное бюджетное учреждение «Национальный медицинский \\ исследовательский центр терапии и профилактической медицины» \\ Министерства здравоохранения Российской Федерации, \\ Россия, 101990, г. Москва, Петроверигский пер., 10, стр. 3 \\ ${ }^{3}$ Белгородский государственный национальный исследовательский университет», \\ Россия, 308015, г. Белгород, Победы, 85 \\ ${ }^{1}$ Voronezh State Medical University named after N.N. Burdenko, \\ 10, Studencheskaya St., Voronezh, 394036, Russia \\ ${ }^{2}$ Federal State Budgetary Institution «National Medical Research Centre for Therapy and Preventive \\ Medicine» of the Ministry of Health of the Russian Federation, \\ 10 Petroverigsky lane, Moscow, 101990, Russia \\ ${ }^{3}$ Belgorod National Research University, \\ 85 Pobedy St., Belgorod, 308015, Russia \\ E-mail: yanavrn@yandex.ru
}

\begin{abstract}
Аннотация
Особый интерес представляет изучение механизмов развития хронической сердечной недостаточности $(\mathrm{XCH})$, особенно с промежуточной фракцией выброса (СНпрФВ) в зависимости от возраста. Методы. Обследовано 80 больных, возраст 55,7 \pm 9,3 лет, СНпрФВ, I-II функциональный класс (ФК) ХСН. Эти больные разделены на 1 группу - среднего возраста (CВ) - 50,2 \pm 4,1 лет (n = 40 чел.); 2 группу - пожилого возраста (ПВ) 60,4 $\pm 3,8$ лет (n= 40 чел.). Результаты. У больных СВ уровень альдостерона (АЛ) был на $23 \%$ выше, чем в КГ $(\mathrm{p}<0,01)$, у больных ПВ - 204,3 $\pm 32,6$ пг/мл, что выше, чем в КГ на $33 \%$ (р < 0,01) и чем у СВ на $14 \%$ $(\mathrm{p}<0,05)$. Нами установлена высокая корреляционная зависимость уровня АЛ от ФК ХСН $\mathrm{r}=0,7819$, (p $<0,001)$. Уровни маркеров баланса коллагена TIMP-1 в группе пациентов СНпрФВ СВ были выше, чем СНпрФВ ПВ (на $26 \%, \mathrm{p}<0,01)$. Уровни ММП-1 в группе пациентов ПВ были выше на $22 \%(\mathrm{p}<0,01)$, чем у пациентов СВ. Уровни ММП-9 в группе ПВ были достоверно выше на $16 \%($ p $<0,01)$, чем в группе пациентов СВ. Нами проведен корреляционный анализ зависимости между ММП-9 и ТІМР-1, выявлена умеренная корреляционная зависимость $(\mathrm{r}=0,65)$ у больных ПВ с СНпрФВ. Вывод: Тяжесть ХСН и возраст увеличивают активацию альдостероновой системы. Показатели АЛ выше у больных СНпрФВ пожилого возраста. У пожилых пациентов имеется деградация внеклеточного матрикса, что подтверждается увеличением ММП-1, ММП-9. Увеличение содержания в крови ТІМР-1 отражает роль фиброобразования в прогрессировании симптомов ХСН. Уровни АЛ, ММП, ТІМР-1 могут быть полезными маркерами для идентификации пациентов с риском прогрессирования СНпрФВ, в том числе в качестве компонентов мультимаркерного подхода.
\end{abstract}




\begin{abstract}
Of particular interest is the study of the mechanisms of development of chronic heart failure (HF), especially with intermediate ejection fraction (HFmrEF) depending on age. Methods. The study involved 80 patients, age 55,7 \pm 9,3 years, HFmrEF, I-II functional class (FC) HF. These patients are divided into: 1 group - middle age $(\mathrm{MA})-50.2 \pm 4.1$ years $(\mathrm{n}=40$ people); 2 group-elderly (E) $60.4 \pm 3.8$ years ( $n=40$ people). Results. In patients with MA, the level of aldosterone (AL) was $23 \%$ higher than in KG ( $\mathrm{p}<0.01$ ), in patients with $\mathrm{E}-204.3 \pm 32.6 \mathrm{PG} / \mathrm{ml}$, which is higher than in $\mathrm{KG}$ by $33 \%(\mathrm{p}<0.01)$ and than in MA by $14 \%(\mathrm{p}<0.05)$. We have established a high correlation between the AL level and the HF FC $r=0.7819$, ( $p<0.001)$. Levels of TIMP-1 collagen balance markers in the group of patients with HFmrEF MA were higher than HFmrEF E (by $26 \%$, p < 0.01). Levels of MMP-1 in the group of E patients were $22 \%$ higher $(p<0.01)$ than in MA patients. MMP-9 levels in the E group were significantly higher by $16 \%(\mathrm{p}<0.01)$ than in the MA group. We performed a correlation analysis of the relationship between MMP-9 and TIMP-1, and found a moderate correlation $(r=0.65)$ in patients with $\mathrm{E}$ with HFmrEF. Conclusion: The severity of HF and age, increase the activation of the aldosterone system. Indicators AL HFmrEF higher in patients of advanced age. Elderly patients have degradation of the extracellular matrix, which is confirmed by an increase in MMP-1, MMP-9. The increase in blood TIMP-1 reflects the role of fibroblast formation in the progression of HF symptoms. Levels of AL, MMP, and TIMP-1 can be useful markers for identifying patients at risk of progression of HFmrEF, including as components of a multi-marker approach.
\end{abstract}

Ключевые слова: хроническая сердечная недостаточность, промежуточная фракция выброса, альдостерон, маркеры фиброза.

Keywords: chronic heart failure middle range ejection fraction, aldosterone, fibrosis markers.

\title{
Введение
}

Сердечная недостаточность является наиболее быстро растущей сердечнососудистой нагрузкой на здоровье во всем мире [Simmonds, 2020]. Прогноз сердечнососудистых заболеваний в значительной степени определяется способностью задерживать или предотвращать развитие и прогрессирование хронической сердечной недостаточности $(\mathrm{XCH})$. Таким образом, внимание смещается на более раннюю диагностику и лечение $\mathrm{XCH}$ [Cleland, 2020]. При этом новые подходы к диагностике и лечению в значительной степени расширяют возможности эффективного контроля клинического состояния пациентов с ХСН. По данным эпидемиологических исследований, за последние годы в мире зарегистрировано увеличение количества пациентов с ХСН. В нашей стране распространенность ХСН в популяции составляет от 7 \% до $10 \%$ [Мареев, 2017], что свидетельствует о необходимости дальнейшего изучения данной проблемы [Вологдина, 2016]. В соответствии с клиническими рекомендациями 2016 года при классификации в зависимости от фракции выброса левого желудочка (ФВ ЛЖ) стали отдельно выделять пациентов с промежуточной (40-49\%) ФВ ЛЖ, процесс накопления данных о которых идет в настоящее время [Клинические рекомендации, 2016; Ponikowski, 2016].

Известно, что развитие сердечно-сосудистых событий при ХСН обязательно сопровождается компенсаторным повышением активности ренин-ангиотензинальдостероновой системы (РААС). РААС проявляет свои эффекты в основном через ангиотензин (АТ) II, который, в свою очередь, стимулирует секрецию альдостерона (АЛ) надпочечниками. В условиях ХCH не только повышается активность РААС, но и многократно возрастает значимость эффектов АЛ - по сравнению с пациентами с нормальной функцией сердца - это и избыточное увеличение внутри- и внесосудистого объема жидкости, развитие фиброза в сердце, почках и других органах, что способствует дальнейшему прогрессированию ХСН [Резник, 2019]. Снижение метаболического клиренса АЛ является дополнительным важным фактором повышения концентрации АЛ в крови больных ХСН. Альдостерон, помимо развития гипокалиемии и гипомагниемии, обладает другими нежелательными эффектами, играющими важную роль в патофизиологии $\mathrm{XCH}$, способствуя 
усилению ремоделирования ЛЖ и коронарных сосудов, эндотелиальной дисфункции, атеросклероза и аритмического синдрома [Pugliese, 2020]. При снижении концентрации АЛ восстанавливается фибринолитическая активность, уменьшается риск дальнейшего тромбообразования и вероятность развития повторного инфаркта. Блокада рецепторов к АЛ сопровождается уменьшением степени фиброза миокарда и риска развития повреждения сосудов, а также улучшением эндотелиальной функции в результате увеличения эндотелиальной биодоступности оксида азота [Münzel, 2015].

Изменение архитектуры и состава экстрацеллюлярного матрикса сердца является ключевым элементом неблагоприятного ремоделирования сердца, возникающего при ХСН. Деструктивные процессы в миокарде могут запускаться различными факторами, которые либо повышают активность матриксных металлопротеиназ (ММП), либо снижают активность их тканевых ингибиторов [Мясоедова, 2016]. В большинстве случаев результатом является развитие фиброза, что оказывает влияние на патофизиологию и клинические исходы ХСН. Накопление фибриллярных коллагенов приводит к развитию фиброза, необратимой дисфункции, что, в свою очередь, является основой для прогрессирования ХСН [Осипова, 2016]. Традиционно считается, что ММП подавляют фиброз из-за их протеолитической активности. Среди ММП непосредственное отношение к ремоделированию миокарда имеют ММП-1 (коллагеназа) и ММП-9 (желатиназа). Ключевую роль в стимуляции накопления фибриллярного внеклеточного матрикса играет ММП-9 [Horn, 2016].

В научных исследованиях представлено незначительное количество работ с комплексным анализом взаимосвязей АЛ с уровнем маркеров фиброза миокарда у пациентов ХСН с промежуточной фракцией выброса ЛЖ.

Цель исследования заключалась в выявлении особенностей влияния альдостерона и маркеров фиброза коллагена на механизмы прогрессирования сердечной недостаточности с промежуточной фракцией выброса у больных в разных возрастных группах.

\section{Материал и методы исследования}

Проведено открытое рандомизированное исследование, в котором приняли участие 80 больных сердечной недостаточностью промежуточной фракцией выброса (СНпрФВ) ишемического генеза I-II функционального класса (ФК) по классификации Нью-Йоркской ассоциации кардиологов (NYHA) (50 мужчин, 30 женщин), средний возраст $55,7 \pm 9,3$ лет. Эти больные в зависимости от возраста были разделены на две группы: 1 группа - в возрасте от 46 до 55 лет $(\mathrm{n}=40$ чел.), средний возраст 50,2 $\pm 4,1 ; 2$ группа - от 56 до 65 лет, средний возраст $-60,4 \pm 3,8$ (n = 40 чел.).

Для определения референтных значений исследуемых показателей нами составлена контрольная группа из 20 здоровых лиц (средний возраст - 52,8 \pm 3,6 лет), у которых по данным клинических и лабораторно-инструментальных исследований отсутствовали признаки ХCH.

Критериями включения больных в исследование считали: 1) ХСН с ФВ ЛЖ 40-49 \% (по Симпсону); 2) I-II ФК по NYHA; 2) верифицированный в стационарных условиях диагноз ИБС, стабильная стенокардия II ФК, 4) перенесённый острый инфаркт миокарда более 12 месяцев назад; 5) подписанное информированное согласие.

Критерии исключения: 1) отказ больного от динамического наблюдения и обследования; 2) сахарный диабет, 3) острые воспалительные, инфекционные, онкологические заболевания; 4) хронические заболевания в стадии обострения; 5) острая и хроническая почечная и печеночная недостаточность; 6) врожденные пороки сердца, кардиомиопатии; 7) злоупотребление алкоголем.

Объективным критерием определения ФК ХСН был тест 6-минутной ходьбы. ХCH I ФК диагностирована у 32 больных (40 \%), у 48 больных (60\%) - ХCH II ФК. 
Эхокардиографию проводили на сканере MyLab 70 (Esaote, Италия) по стандартному протоколу.

Методом иммуноферментного анализа по методикам, рекомендованным производителями реактивов, определяли уровень биохимических маркеров: ММП-1 типа (нг/мл) и ММП-9 типа (нг/мл) с помощью коммерческой тест-системы «MMP-1 ELISA» («Bender Medsystems», Австрия); тканевого ингибитора металлопротеиназы-1 (нг/мл) с помощью коммерческой тест - системы «Human TIMP-1 ELISA» («Bender Medsystems», Австрия); АЛ с помощью коммерческой тест - системы («Aldosteron EIA, Diagnostic Sistems Laboratories», Inc. США).

Непрерывные количественные значения выражали как среднее \pm SD или медиана (Me) и $25 \%-75 \%$ интерквартильный размах. Корреляции между двумя переменными оценивались с помощью рангового критерия Спирмена. Сравнение количественных переменных при нормальном распределении признака проводили с помощью t-критерия Стьюдента, достоверными считали различия при уровне значимости $\mathrm{p}<0,05$.

\section{Результаты и их обсуждение}

Одной из главных составляющих нейрогуморальных изменений, влияющих на прогрессирование ХCH и ремоделирование миокарда, является состояние РАAC, о которой судят по содержанию в периферической венозной крови АЛ [Осипова, 2015]. В связи с этим, нами изучено содержание в крови АЛ у больных СНпФВ, ИБС в зависимости от возраста, а также в зависимости от ФК по NYHA. Результаты представлены в таблице 1.

Таблица 1

Table 1

Содержание в крови альдостерона в исследуемых группах больных СНпрФВ, ИБС в разных возрастных группах $(\mathrm{M} \pm \mathrm{SD})$

The blood content of aldosterone in the studied groups of patients HFmrEF, CHD in different age groups $(\mathrm{M} \pm \mathrm{SD})$

\begin{tabular}{|l|c|}
\hline \multicolumn{1}{|c|}{ Группы больных } & Показатели \\
\cline { 2 - 2 } & Альдостерон, пг/мл \\
\hline Общая группа больных СНпрФВ ИБС $(\mathrm{n}=80)$ & $195,2 \pm 35,1^{\Delta}$ \\
\hline СНпрФВ, ИБС пожилой возраст $(\mathrm{n}=40)$ & $204,3 \pm 32,6^{*} \#$ \\
\hline СНпрФВ, ИБС средний возраст $(\mathrm{n}=40)$ & $175,4 \pm 41,1^{*}$ \\
\hline Контрольная группа $(\mathrm{n}=20)$ & $135,9 \pm 34,7$ \\
\hline
\end{tabular}

Примечание: ${ }^{\wedge} \mathrm{p}<0,01$ - различия между общей группой больных СНпрФВ ИБС и КГ; *p $<$ 0,05 - различия между группой СНпрФВ, ИБС среднего возраста, группой СНпрФВ ИБС пожилого возраста и КГ; \# $\mathrm{p}<0,01$ - различия между группами СНпрФВ, ИБС среднего и СНпрФВ ИБС пожилого возраста.

Так, в общей группе больных СНпрФВ ишемического генеза установлен уровень АЛ 195,2 \pm 35,1 пг/мл, что было на 30 \% выше по сравнению с КГ, в которой его содержание составило $135,9 \pm 34,7$ пг/мл ( $<0,01)$. Сравнительный анализ групп СНпрФВ на фоне ИБС в зависимости от возраста показал (рис. 1), что у больных СНпрФВ среднего возраста (CB) уровень АЛ составил $175,4 \pm 41,1$ пг/мл, что превышало на $23 \%$ показатели в КГ $(\mathrm{p}<0,01)$. У больных СНпрФВ пожилого возраста (ПВ) значимо усилен синтез АЛ - они имели достоверно более высокие показатели - 204,3 \pm 32,6 пг/мл, что превышало уровень АЛ пациентов КГ на $33 \%(135,9 \pm 34,7)(\mathrm{p}<0,01)$ и достоверно отличалось от показателей группы СНпрФВ СВ на $14 \%(\mathrm{p}<0,05)(175,4 \pm 41,1$ пг/мл). 


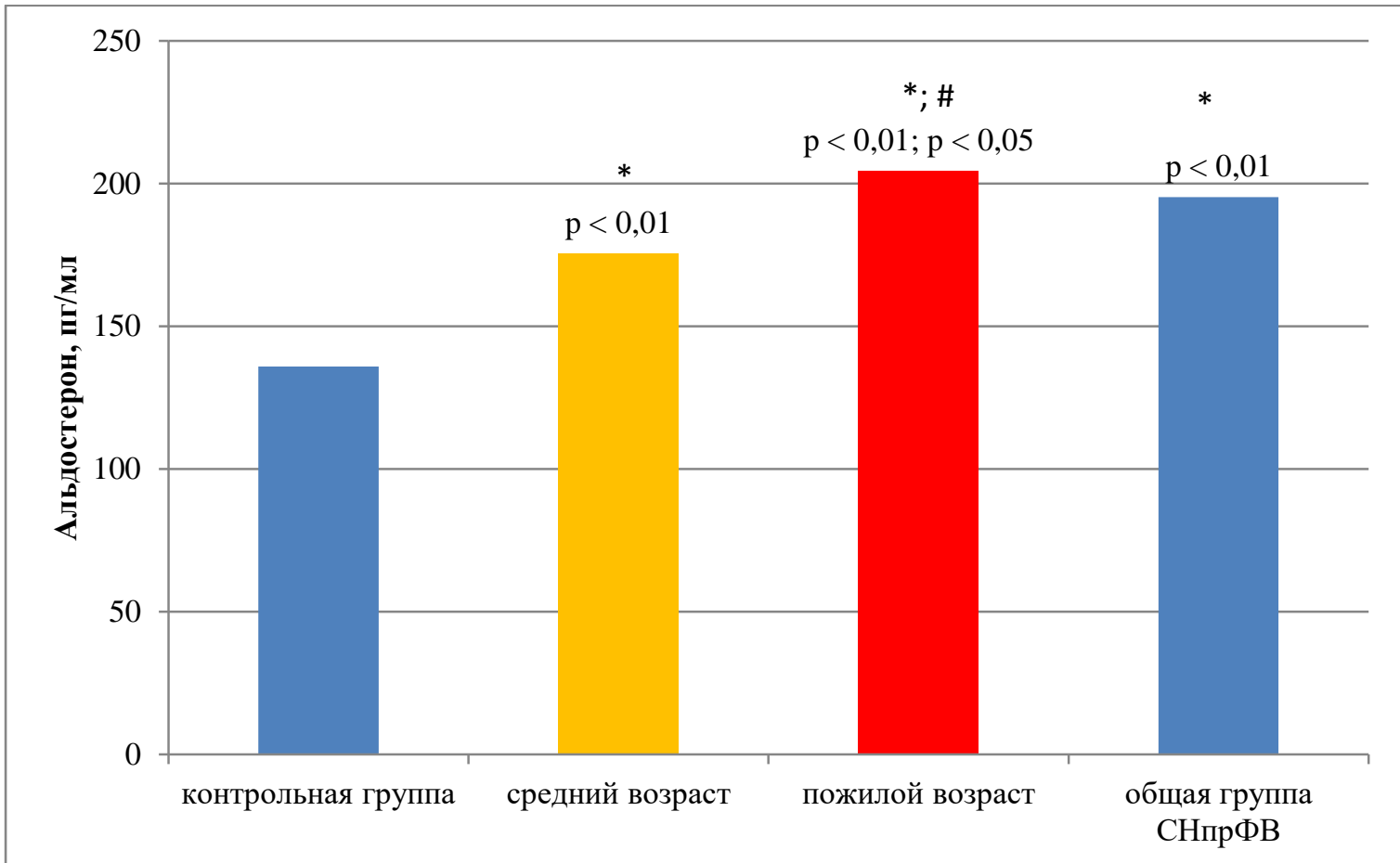

Рис. 1. Уровень альдостерона в группах СНпрФВ в зависимости от возраста. $*-$ p $<0,01-$ разница между КГ и группами СВ и ПВ и общей группой; \#р < 0,05 - между больными СВ и ПВ

Fig. 1. The level of aldosterone in groups HFmrEF depending on age. $*-p<0.01$ difference between the control group and the middle-aged and elderly groups and the General group; \#p $<0.05$ - between middle-aged and elderly patients

Таким образом, можно утверждать, что с возрастом ухудшается течение нейрогормональных показателей и тем самым оказывают дополнительное влияние на фиброзное изменение структурно-функциональные свойства сердца. В связи с чем особый интерес представляет изучение содержания АЛ в зависимости от тяжести течения ХСН у больных разных возрастных групп.

Таблица 2

Table 2

Содержание в крови альдостерона в изучаемых группах больных СНпрФВ, ИБС в зависимости от ФК XCH $(\mathrm{M} \pm \mathrm{SD})$

Blood levels of aldosterone in the studied groups of patients HFmrEF, CHD depending on the functional class of $\mathrm{HF}(\mathrm{M} \pm \mathrm{SD})$

\begin{tabular}{|c|c|}
\hline Группа & $\begin{array}{c}\text { Показатели } \\
\text { Альдостерон, пг/мл }\end{array}$ \\
\hline \multicolumn{2}{|c|}{ Общая группа больных СНпрФВ, ИБС (n = 80) } \\
\hline & $195,2 \pm 35,1^{\Delta}$ \\
\hline \multicolumn{2}{|c|}{ СНпрФВ, ИБС ПВ (n=40) } \\
\hline I ФК, $\mathrm{n}=10$ чел & $182,3 \pm 33,1$ \\
\hline II ФК, $\mathrm{n}=30$ чел & $219,7 \pm 31,2 *$ \\
\hline \multicolumn{2}{|c|}{ СНпрФВ, ИБС СВ (n=40) } \\
\hline I ФК, $\mathrm{n}=22$ чел & $163,1 \pm 40,3 \#$ \\
\hline II ФК, $\mathrm{n}=18$ чел & $186,5 \pm 41,9 * \wedge$ \\
\hline Контрольная группа $\mathrm{n}=20$ че. & $135,9 \pm 34,7$ \\
\hline
\end{tabular}

Примечание: ${ }^{\Delta} \mathrm{p}<0,01$ - различия между общей группой больных СНпрФВ ИБС и КГ; *p < 0,05 - различия между больными СНпрФВ I ФК и II ФК; \#p - * < 0,05 - различия между больными СНпрФВ I ФК ПВ и СВ ; ^p < 0,05 различия между больными СНпрФВ II ФК ПВ и СВ. 
При сравнительном анализе изменений уровня АЛ у больных СНпрФВ в зависимости от тяжести ХСН установлено, что в общей группе уровень АЛ составил $195,2 \pm 35,1$ пг/мл, что на 30 \% превышает показатели КГ 135,9 $\pm 34,7$ пг/мл (p < 0,01).

У больных ІІ ФК ПВ содержание в крови АЛ было на $17 \%$ выше, чем у больных I ФК (p < 0,05) $(219,7 \pm 31,2$ и 182,3 $\pm 33,1$ пг/мл соответственно). У больных СНпрФВ СВ, имеющих II ФК, содержание АЛ было 186,5 \pm 41,9 пг/мл, что также достоверно выше на $13 \%$ по отношению к показателям больных I ФК 163,1 \pm 40,3 (p < 0,05) .

При этом интерес представляют данные, что тяжесть ХСН и возраст увеличивают активацию альдостероновой системы, и в нашем исследовании доказано, что самые высокие показатели АЛ были у больных ІІ ФК СНпрФВ ПВ. Так, у больных ІІ ФК ПВ содержание в крови АЛ было на $15 \%$ выше, чем у больных СВ $(\mathrm{p}<0,05)$. У пожилых больных I ФК содержание АЛ было также выше на $11 \%$ по сравнению с больными СВ $(\mathrm{p}<0,05)$. В нашей работе установлена высокая корреляционная зависимость уровня АЛ от тяжести ХCH r $=0,7819,(\mathrm{p}<0,001)$ у больных ПВ (рис. 2).

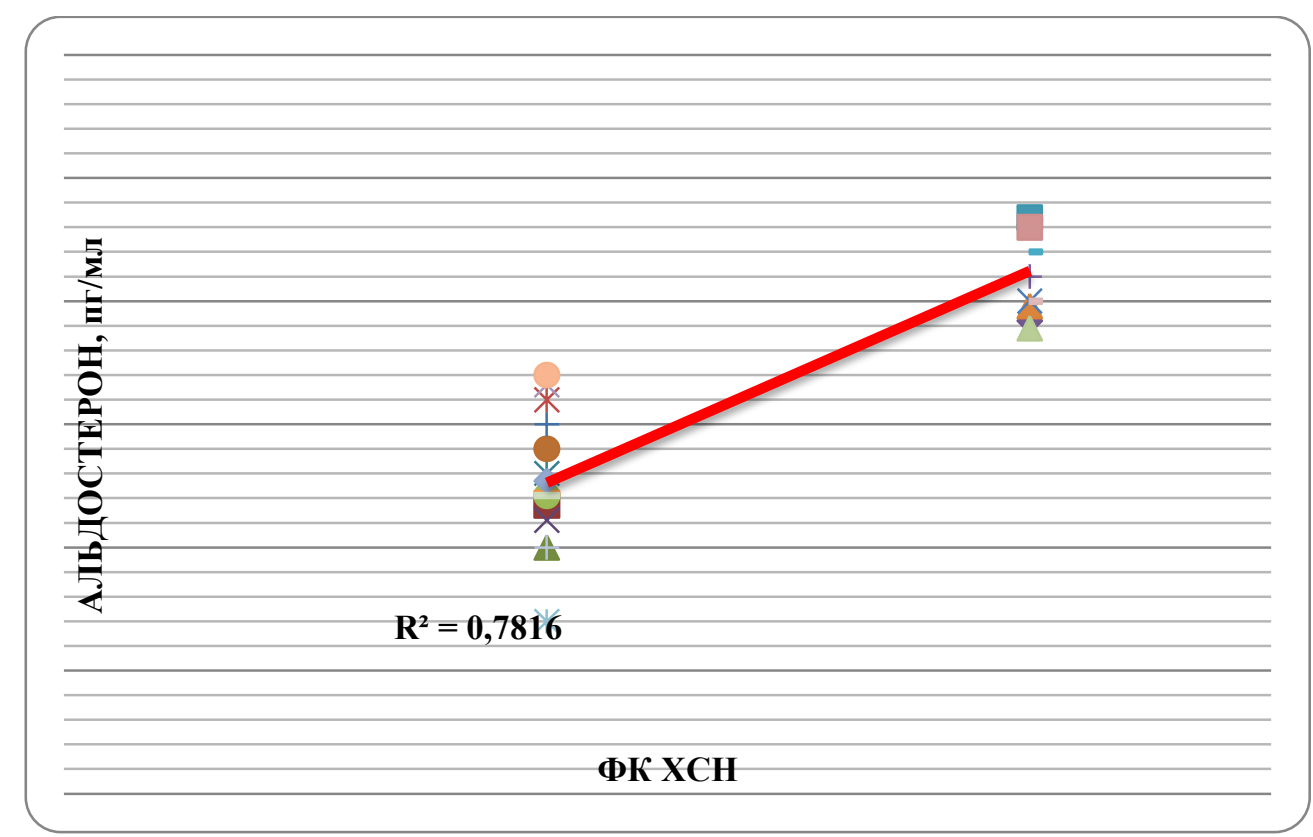

Рис. 2. Корреляционная зависимость уровня АЛ с ФК ХСН

Fig. 2. Correlation of aldosterone levels with the severity of HF

Таким образом, РААС является центральным регулятором сердечно-сосудистой системы и играет важную роль в патофизиологии $\mathrm{XCH}$, характеризующейся нейроэндокринной активацией. АЛ повышает эффекты АТ II за счет увеличения транскрипции АТ-1-рецепторов и ангиотензинпревращающего фермента, а АТ-II, в свою очередь, увеличивает содержание АЛ. АЛ индуцирует клеточную пролиферацию и запускает каскад синтеза коллагена с образованием фиброзной ткани и активирует фибробласты путем активации минералокортикоидных рецепторов [Драпкина, 2012].

Известно, что при ХСН происходит регуляция содержания ММП, которые оказывают влияние на ремоделирование желудочков [Sanchis, 2015].

Росту белков внеклеточного матрикса способствует MMP-1, прогрессированию коронарного атеросклероза - ММР-9, они изменяют экстрацеллюлярный матрикс миокарда и тем самым способствуют неблагоприятному ремоделированию желудочков при ХCH [Burke, 2019]. Повышенные уровни ММП-9 в плазме крови коррелируют с увеличением объема ЛЖ и снижением ФВ при XСН [Kunkel, 2019]. Избыточное развитие фиброза в миокарде происходит вследствие потери физиологической реципрокной 
регуляции между факторами, влияющими на синтез и деструкцию фибриллярного коллагена.

В здоровом сердце экспрессия и функции ММП жестко регулируются. В патологии активность ММП увеличивается, что приводит к дисбалансу между синтезом и деградацией внеклеточного матрикса, развитию фиброза в миокарде. Это влечет за собой значительное изменение синтеза внеклеточного матрикса, повышение механической жесткости миокарда и способствует возникновению диастолической дисфункции.

Однако взаимосвязь между ММП-1, ММП-9 и тканевыми ингибиторами ММП-1 (ТIMP-1) не изучена у больных СНпрФВ в разных возрастных категориях.

В связи с этим нами изучены показатели фиброза у больных СНпрФВ ишемического генеза в зависимости от возраста и ФК ХСН.

У пациентов с СНпрФВ наблюдалось усиление как процессов образования, так и деградации интерстициального коллагена, но в зависимости от возраста имелись различия по изучаемым показателям, которые представлены в таблице 3.

Таблица 3

Table 3

Уровень маркеров баланса коллагена у пациентов с СНпрФВ в зависимости от возраста $\mathrm{Me}\left(\mathrm{Q}_{25} ; \mathrm{Q}_{75}\right)$

The levels of markers of collagen balance in patients with HFmrEF depending on age $\mathrm{Me}\left(\mathrm{Q}_{25} ; \mathrm{Q}_{75}\right)$

\begin{tabular}{|c|c|c|c|}
\hline \multirow{2}{*}{ Показатели/Группа } & $\begin{array}{c}\text { СНпрФВ, ИБС ПВ } \\
\mathrm{n}=40\end{array}$ & $\begin{array}{c}\text { СНпФВ, ИБС СВ } \\
\mathrm{n}=40\end{array}$ & КГ, $\mathrm{n}=20$ \\
\hline \multirow{2}{*}{ ММП-1, нг/мЛ } & 8,2 & 6,4 & 3,0 \\
& $(4,51 ; 12,74)^{\Delta_{*}}$ & $(3,42 ; 8,61)^{\Delta}$ & $(2,21 ; 4,05)$ \\
\hline \multirow{2}{*}{ ММП-9, нг/мл } & 152 & 128 & 36 \\
& $(131 ; 181)^{\Delta_{*}}$ & $(109 ; 163)^{\Delta}$ & $(28 ; 46)$ \\
\hline \multirow{2}{*}{ ТІМР-1, нг/мл } & 216 & 293 & 175 \\
& $(159 ; 311)^{\Delta *}$ & $(167 ; 349)^{\Delta}$ & $(155 ; 245)$ \\
\hline \multirow{2}{*}{ ММП-9/ТІМР-1 } & 0,70 & 0,47 & 0,21 \\
& $(0,51 ; 0,82)^{\Delta_{*}}$ & $(0,34 ; 0,53)^{\Delta}$ & $(0,19 ; 0,25)$ \\
\hline
\end{tabular}
ИБС СВ.

Примечание: $\Delta \mathrm{p}<0,01$ - по сравнению с КГ; * $\mathrm{p}<0,01$ - с группой пациентов с СНпФВ,

Оценивая полученные результаты, можно констатировать, что уровень ТIMP-1 в группе больных СНпрФВ, ИБС СВ (на $40 \%, \mathrm{p}<0,01)$ и ПВ (на $19 \%, \mathrm{p}<0,01)$ был достоверно выше, чем в КГ. При этом уровни маркеров баланса коллагена TIMP-1 в группе пациентов СВ были выше, чем у пациентов ПВ (на $26 \%$, p < 0,01). Уровни маркеров деградации интерстициального коллагена (ММП-1) в группе пациентов ПВ были достоверно выше на $22 \%(\mathrm{p}<0,01)$, чем в группе CВ, на $63 \%(\mathrm{p}<0,001)$ выше, чем в КГ. В группе CВ данный показатель был на $53 \%$ выше $(\mathrm{p}<0,001)$, чем в КГ. Уровни маркеров деградации интерстициального коллагена (ММП-9) в группе ПВ были достоверно выше на $16 \%$ $(\mathrm{p}<0,01)$, чем в группе CВ, и на $76 \%(\mathrm{p}<0,001)$ выше, чем в КГ. В группе СВ данный показатель был на $72 \%$ выше $(\mathrm{p}<0,001)$, чем в КГ.

Наше исследование показало, что у пациентов ПВ соотношение ММП-9/ТІМР-1 было максимальным (0,70), у пациентов СВ соотношение ниже - 0,47 (на $33 \%, p<0,01$ ). По сравнению с КГ (соотношение 0,21$)$ разница у больных ПВ составила $70 \%$ (p < 0,01), в группе CВ - 55 \% (p < 0,01). Дисбаланс в соотношении ММП-9/ТIMР-1 отражает прогрессирующее ремоделирование желудочков.

Таким образом, проанализировав уровень маркеров баланса коллагена у пациентов с СНпрФВ в зависимости от возраста, можно сделать вывод о патогенетической роли постарения больных в дисбалансе обмена коллагена при ХСН. 
Так, у пациентов с СНпрФВ СВ четко прослеживалось усиление процессов фиброза и образования коллагена I типа, о чем свидетельствовало увеличение уровня TIMP-1 по сравнению с КГ, и одновременное увеличение деструкции компонентов внеклеточного матрикса, на что указывало увеличение уровня ММП-1 по сравнению с КГ. У пациентов ПВ отмечалось более выраженное увеличение ММП-1 и ММП-9 при снижении уровня TIMP-1.

В связи с этим интерес представляет изучение содержания маркеров фиброза коллагена в зависимости от ФК ХСН у больных в изучаемых возрастных группах. Результаты представлены в таблице 4.

Таблица 4

Table 4

Уровень маркеров баланса коллагена у пациентов с СНпФВ изучаемых групп в зависимости от $\Phi К ~ X C H$

The level of markers of collagen balance in patients with HFmrEF in the study groups depending on HF by NYHA

\begin{tabular}{|c|c|c|c|c|c|}
\hline Группа & ФК по NYHA & МПП-1 нг/мЛ & $\begin{array}{c}\text { МПП-9 } \\
\mathrm{HГ} / \mathrm{MЛ}\end{array}$ & $\begin{array}{c}\text { TIMP-1 } \\
\text { нг/мл }\end{array}$ & $\begin{array}{l}\text { МПП-9/ } \\
\text { TIMР-1 }\end{array}$ \\
\hline \multirow{2}{*}{$\begin{array}{l}\text { СНпФВ, ИБС, } \\
\text { ПВ }\end{array}$} & $\mathrm{I}(\mathrm{n}=10$, чел $)$ & $\begin{array}{c}6,5 \\
(4,74 ; 7,81) \#\end{array}$ & $\begin{array}{c}146 \\
(131 ; 158) \#\end{array}$ & $\begin{array}{c}270 \\
(223 ; 311) \#\end{array}$ & $\begin{array}{c}0,54 \\
(0,51 ; 0,59) \#\end{array}$ \\
\hline & II (n = 30, чел) & $\begin{array}{c}8,9 \\
(6,93 ; 12,74) * \Delta\end{array}$ & $\begin{array}{c}158 \\
(132 ; 181)^{* \Delta}\end{array}$ & $\begin{array}{c}208 \\
(159 ; 221)^{*}\end{array}$ & $\begin{array}{c}0,75^{*}(0,70 \\
0,82)^{* \Delta}\end{array}$ \\
\hline \multirow{2}{*}{$\begin{array}{l}\text { СНпФВ, ИБС, } \\
\text { СВ }\end{array}$} & I (n = 22, чел) & $\begin{array}{c}5,6 \\
(3,42 ; 6,14)\end{array}$ & $\begin{array}{c}120 \\
(109 ; 142) \\
\end{array}$ & $\begin{array}{c}306 \\
(284 ; 349) \\
\end{array}$ & $\begin{array}{c}0,39 \\
(0,34 ; 0,45)\end{array}$ \\
\hline & II (n = 18, чел) & $\begin{array}{c}7,4 \\
(7,20 ; 8,61)^{*}\end{array}$ & $\begin{array}{c}132 \\
(121 ; 163)^{*}\end{array}$ & $\begin{array}{c}274 \\
(167 ; 226)^{*} \\
\end{array}$ & $\begin{array}{c}0,48 \\
(0,45 ; 0,53)^{*} \\
\end{array}$ \\
\hline КГ & $0(\mathrm{n}=20$, чел) & $\begin{array}{c}3,0 \\
(2,21 ; 4,05)\end{array}$ & $\begin{array}{c}36 \\
(27,21 ; 44,85)\end{array}$ & $\begin{array}{c}175 \\
(155 ; 192)\end{array}$ & $\begin{array}{c}0,21 \\
(0,19 ; 0,25) \\
\end{array}$ \\
\hline
\end{tabular}

Примечание: *p < 0,05 - различия между больными I ФК и II ФК; \#p - * < 0,05 - различия между больными I ФК ПВ и СВ; ${ }^{\Delta} \mathrm{p}<0,05$ различия между больными II ФК ПВ и СВ.

При анализе полученных результатов в зависимости от ФК ХСН в группе больных ПВ II ФК нами выявлено увеличение на $27 \%(\mathrm{p}<0,01)$ содержания в крови ММП-1 (рис. 3a) по сравнению с больными I ФК, по содержанию в крови ММП-9 (рис. 3b) разница составила $8 \%(\mathrm{p}<0,05)$. У больных II ФК уровень ТІМР-1 (рис. $3 \mathrm{c})$ был на $23 \%$ ниже $(\mathrm{p}<0,01)$, чем у ПВ при I ФК ХСН. Соотношение МПП-9/ТIMP-1 (рис. 3d) у больныХ II ФК было на $72 \%$ выше $(\mathrm{p}<0,001)$, чем при I ФК.

В группе больных СВ II ФК нами выявлено увеличение на $24 \%(\mathrm{p}<0,01)$ содержания в крови ММП-1 по сравнению с больными I ФК, по содержанию в крови ММП-9 разница составила $9 \%(\mathrm{p}<0,05)$. У больных II ФК уровень ТІМР-1 был на $10 \%$ ниже $(\mathrm{p}<0,05)$, чем у больных СВ при I ФК ХCH. Соотношение МПП-9/ТIMP-1 у больных II ФК было на 19 \% выше (p <0,01), чем при I ФК.

Нами проанализированы маркеры фиброза в зависимости от возраста в подгруппах соответствующих ФК. Так, у больных ПВ с I ФК уровень ММП-1 был на 14 \% выше $(\mathrm{p}<0,05)$, чем у больных I ФК СВ, а при II ФК разница составила $17 \%(\mathrm{p}<0,01)$. Уровень ММП-9 больных с I ФК был на $18 \%$ выше $(\mathrm{p}<0,01)$, чем у больных I ФК СВ, а при II ФК разница составила $16 \%(\mathrm{p}<0,01)$. Нами также проанализирована динамика TIMP-1 в зависимости от возраста. Так, у больных ПВ I ФК уровень ТІМР-1 был на $12 \%$ ниже $(\mathrm{p}<0,05)$, чем у больных I ФК СВ, а при II ФК разница составила $24 \%$ $(\mathrm{p}<0,01)$. У больных І ФК ПВ соотношение МПП-9/ТІМР-1 было на $28 \%$ выше $(\mathrm{p}<0,01)$, чем при I ФК у больных $\mathrm{CB}$, а у больных со II ФК ХCH разница между группами составила $36 \%$. 

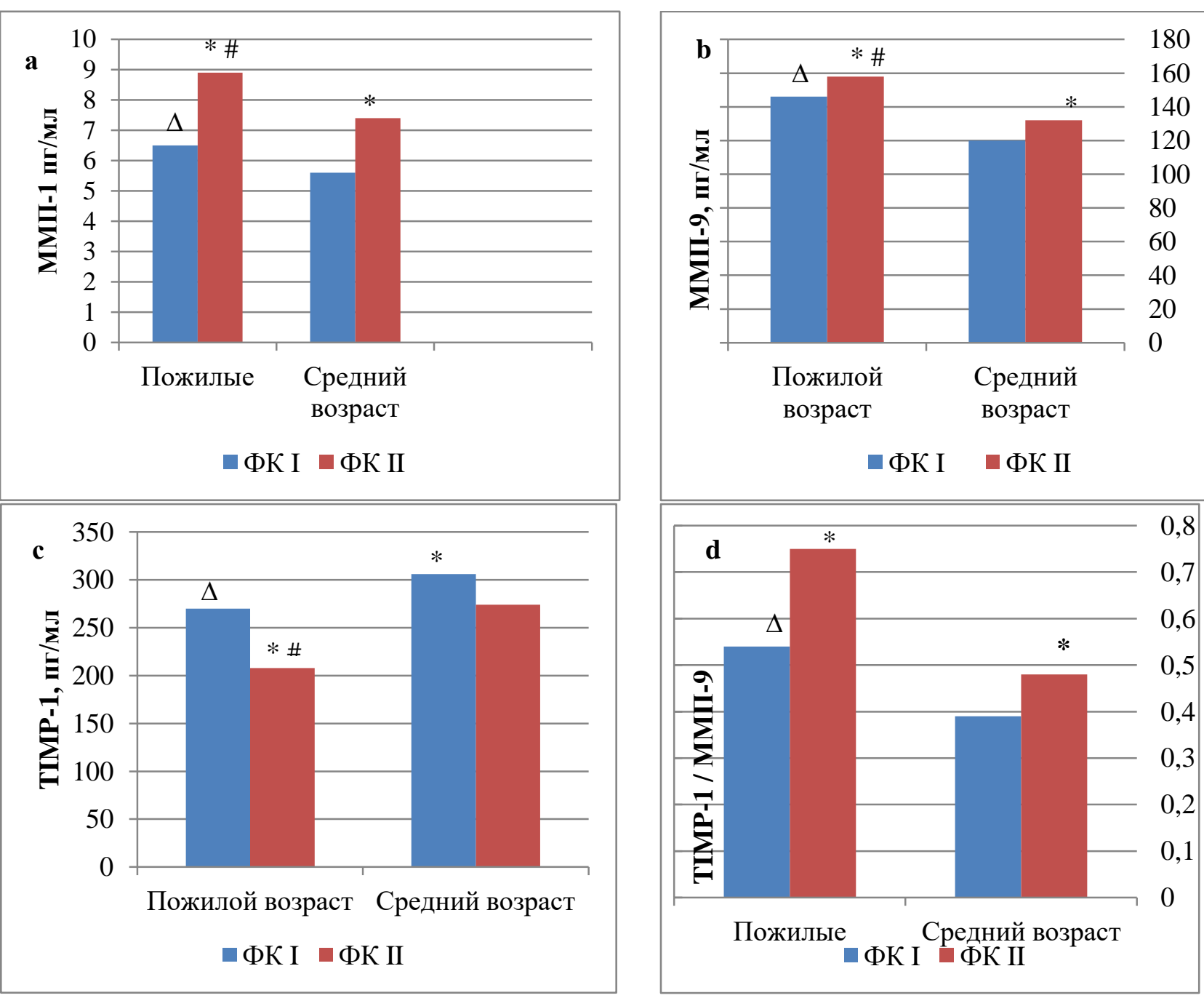

Рис. 3. Уровни ММП-1 (a), ММП-9 (b), ТІМР-1 (c), ММП-9/ТІМР-1 (d) в зависимости от возраста. *p $<0,01$ - разница между группой ФК I и группой ФК II СВ и ПВ; \# - p < 0,05 - между больными

СВ и ПВ ФК II; $\Delta \mathrm{p}<0,05$ - между больными среднего и пожилого возраста ФК I

Fig. 3. Levels of MMP-1 (a), MMP-9 (b), TIMP-1 (c), MMP-9/TIMP-1 (d) in HFmrEF groups depending on age. ${ }^{*} p<0.01$ - difference between the HF I group and the HF II group of middle - aged and elderly; $\#-\mathrm{p}<0.05$ - between middle-aged and elderly HF II patients; $\Delta \mathrm{p}<0.05$ - between middle-aged and elderly HF I patients

Нами проведен корреляционный анализ зависимости между ММП-9 и ТIMP-1 (рис. 4). Выявлена умеренная корреляционная зависимость $(\mathrm{r}=0,65)$ между изучаемыми показателями у больных ПВ СНпрФВ.

С современной точки зрения, основным маркером ХСН является именно фиброз, который и определяет наличие диастолической дисфункции, морфологической гетерогенности миокарда, склонности к прогрессированию ХСН [Попов, 2019].

Изменение архитектуры и состава экстрацеллюлярного матрикса, развитие фиброза является ключевым элементом неблагоприятного ремоделирования сердца, возникающего при ХСН. Проведенные исследования доказали, что состояние активного ремоделирования миокарда сопровождается усиленной активацией ММП-9 и, соответственно, увеличением их циркулирующих концентраций [Radosinska, 2017]. Bce больше данных свидетельствуют о том, что биомаркеры играют роль в диагностике XCH [Ferreir, 2017]. 


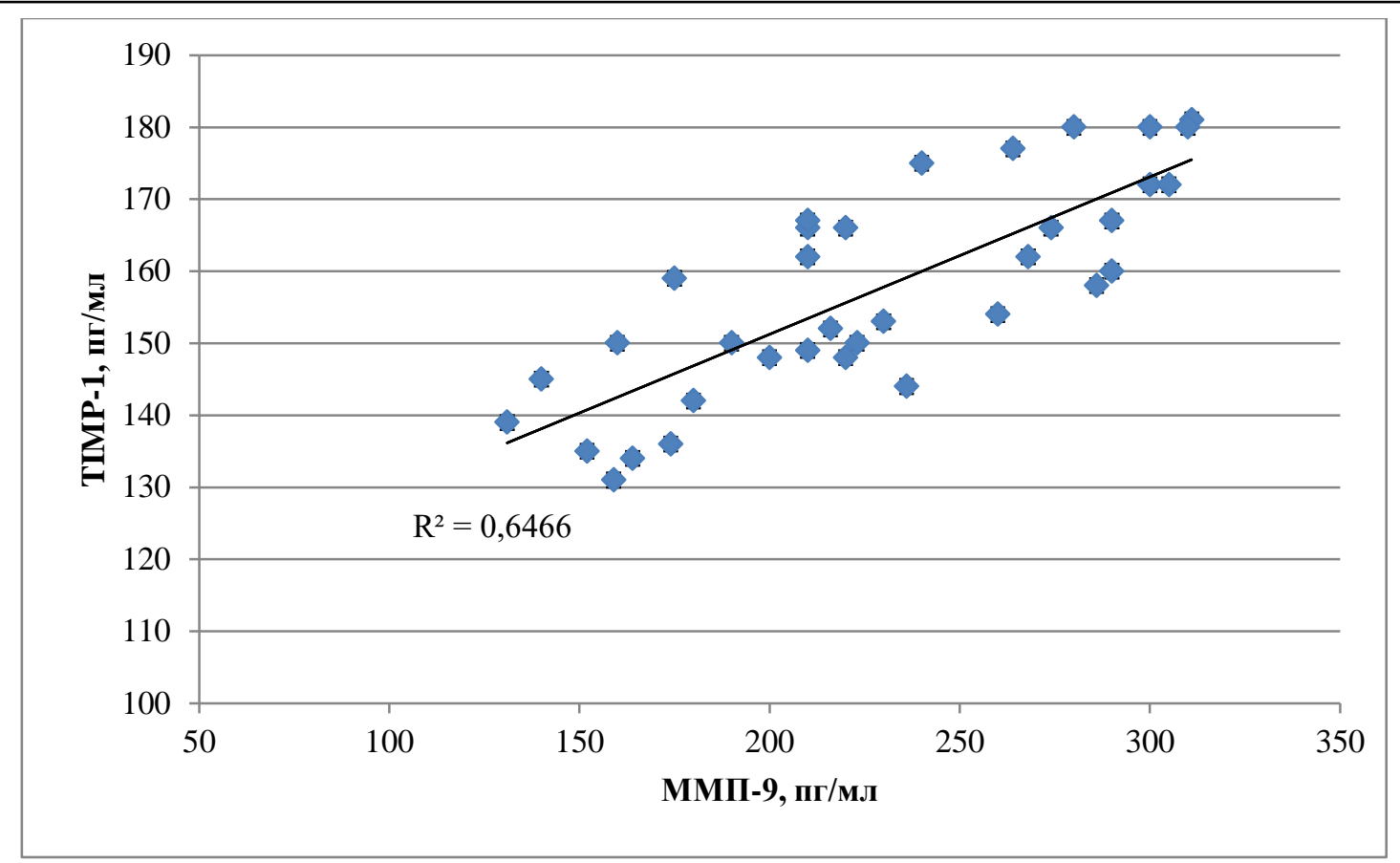

Рис. 4. Корреляционная зависимость уровня ММП-9 с ТІМР-1 у больных с СНпФВ ПВ

Fig. 4. Correlation between the level of MMP-9 and TIMP-1 in elderly patients with HFmrEF

На сегодняшний день доказано, что клетки эндотелия, кардиомиоциты и другие могут изменять процесс фиброзообразования через синтез протеаз, участвующих в метаболизме экстрацеллюлярного матрикса, и секрецию матриксных белков в зависимости от вида повреждения миокарда [Toba, 2019]. Избыточное развитие фиброза в миокарде происходит вследствие нарушения физиологического соотношения между факторами, влияющими на синтез и деструкцию фибриллярного коллагена.

Наше исследование показало, что у пациентов ПВ с СНпрФВ соотношение ММП-9/ТIMР-1 были выше по сравнению с больными СВ, что согласуется с данными литературы, которые демонстрируют ассоциацию между ММП-9 и ухудшением насосной у пациентов ХСН, отражая прогрессирующее ремоделирование желудочков [Morishita, 2017].

\section{Заключение}

Таким образом, АЛ индуцирует клеточную пролиферацию и запускает каскад синтеза коллагена с образованием фиброзной ткани. В нашем исследовании доказано, что тяжесть ХСН и возраст увеличивают активацию альдостероновой системы. Самые высокие показатели АЛ нами выявлены у больных ІІ ФК СНпрФВ ПВ.

Нами установлена активизация процессов деградации внеклеточного матрикса, особенно выраженная в группе больных СНпрФВ ПВ, что подтверждается увеличением ММП-1, ММП-9. Увеличение же содержания в крови ТIMР-1 отражает патогенетическую роль процессов фиброзирования в прогрессировании симптомов при более тяжелом ФК ХСН.

Уровни АЛ, ММП, ТІМР-1 могут быть полезными маркерами для идентификации пациентов с риском прогрессирования СНпрФВ, в том числе в качестве компонентов мультимаркерного подхода.

\section{Список литературы}

1. Вологдина И.В., Симаненков В.И., Порошина Е.Г., Минько Б.А. 2016. Качество жизни и приверженность терапии у пациентов пожилого и старческого возраста с хронической сердечной недостаточностью, коморбидными когнитивными и аффективными нарушениями. Вестник Северо-Западного государственного медицинского университета им. И.И. Мечникова, 1 (8): 45-49. 
2. Драпкина О.М., Драпкина Ю.С. 2012. Фиброз и активность ренин-ангиотензинальдостероновой системы. Реалии и перспективы. Артериальная гипертензия, 18 (5): 449-458.

3. Клинические рекомендации. 2016. Хроническая сердечная недостаточность (ХCH). Общество специалистов по сердечной недостаточности и Российское кардиологическое общество. $92 \mathrm{c}$.

4. Мареев В.Ю., Фомин И.В., Агеев Ф.Т., Арутюнов Г.П., Беграмбекова Ю.Л., Беленков Ю.Н., Васюк Ю.А., Галявич А.С., Гарганеева А.А., Гендлин Г.Е., Гиляревский С.Р., Глезер М.Г., Драпкина О.М., Дупляков Д.В., Кобалава Ж.Д., Козиолова Н.А., Лопатин Ю.М., Мареев Ю.В., Моисеев В.С., Недошивин А.О., Перепеч Н.Б., Ситникова М.Ю., Скибицкий В.В., Тарловская Е.И., Чесникова А.И., Шляхто Е.В. 2017. Клинические рекомендации. Хроническая сердечная недостаточность $(\mathrm{XCH})$. Сердечная Недостаточность, 18 (3): 3-40. DOI: 10.18087/rhfj.2017.1.2346.

5. Мясоедова Е.И. 2016. Содержание матриксной металлопротеиназы-1 и ее ингибитора у пациентов с ишемической кардиомиопатией. Вестник новых медицинских технологий, 23 (4): 50-53. DOI: $10.12737 / 23850$.

6. Осипова О.А., Нагибина А.И., Комисов А.А., Петрова Г.Д., Шеховцова Л.В., Власенко М.А., Власенко О.А. 2016 Патоморфологические механизмы регуляции образования миокардиального фиброза у больных хронической сердечной недостаточностью на фоне ишемической болезни сердца. Журнал сердечная недостаточность, 17 (5): 357-364. DOI: 10.18087/RHFJ.2016.5.2137.

7. Осипова О.А., Никитина В.В., Шеховцова Л.В., Нагибина А.И., Власенко М.А. 2015. Нейрогуморальные модуляторы в лечении больных с хронической сердечной недостаточностью. Фундаментальные исследования. 1(8): 1655-1658.

8. Попов М.А., Шумаков Д.В., Зыбин Д.И., Гуревич Л.Е., Ашевская В.Е., Бабокин В.Е., Пронина В.П. 2019. Феномен роли коллагена IV типа и матриксной металлопротеиназы-9 типа в ремоделировании миокарда левого желудочка при ишемической болезни сердца. Российский кардиологический журнал, 24 (8): 83-87. DOI: 10.15829/1560-4071-2019-8-83-87.

9. Резник Е.В., Никитин И.Г. 2019. Кардиоренальный синдром у больных с сердечной недостаточностью как этап кардиоренального континуума (часть I): определение, классификация, патогенез, диагностика, эпидемиология (обзор литературы). Архивъ внутренней медицины, 9 (1): 5-22. DOI: 10.20514/2226-6704-2019-9-1-5-22.

10. Burke R.M., Lighthouse J.K., Mickelsen D.M., Small E.M. 2019. Sacubitril/Valsartan Decreases Cardiac Fibrosis in Left Ventricle Pressure Overload by Restoring PKG Signaling in Cardiac Fibroblasts. Circ. Heart. Fail., 12 (4): e005565. DOI: 10.1161/CIRCHEARTFAILURE.118.005565.

11. Cleland J.G.F., Lyon A.R., Mcdonahh T., McMurray J.J.V., 2020. The year in cardiology: the year in cardiology 2019, European Heart Journal, 41 (12): 1232-1248, https://doi.org/10.1093/eurheartj/ehz949.

12. Ferreira J.M., Ferreira S.M., Ferreira M.J., Falcão-Pires I. 2017. Circulating Biomarkers of Collagen Metabolism and Prognosis of Heart Failure with Reduced or Mid-Range Ejection Fraction. Curr. Pharm. Des., 23 (22): 3217-3223. DOI: 10.2174/1381612823666170317124125.

13. Horn M.A, Trafford A.W. 2016. Aging and the cardiac collagen matrix: Novel mediators of fibrotic remodelling. Journal of Molecular and Cellular Cardiology, 93, 175-185. DOI: 10.1016 / j. yjmcc.2015.11.005.

14. Kunkel G.H., Kunkel C.J., Ozuna H., Miralda I., Tyagi S.C. 2019. TFAM overexpression reduces pathological cardiac remodeling. Molecular and cellular biochemistry, 454 (1-2), 139-152. https://doi.org/10.1007/s11010-018-3459-9.

15. Morishita T., Uzui H., Mitsuke Ya., Amaya N., Kaseno K., Ishida K. 2017. Association between matrix metalloproteinase-9 and worsening heart failure events in patients with chronic heart failure. ESC Heart Failure; 4: 321-330. DOI: 10.1002/ehf2.12137.

16. Münzel T., Gori T., Keaney J.F.Jr., Maack C., Daiber A. 2015. Pathophysiological role of oxidative stress in systolic and diastolic heart failure and its therapeutic implications. European heart journal, 36(38): 2555-2564. https://doi.org/10.1093/eurheartj/ehv305.

17. Ponikowski P., Voors A.A., Anker S.D., Bueno H., Cleland J.G., Coats A.J., Falk V., González-Juanatey J.R., Harjola V.P., Jankowska E.A., Jessup M., Linde C., Nihoyannopoulos P., Parissis J.T., Pieske B., Riley J.P., Rosano G.M., Ruilope L.M., Ruschitzka F., Rutten F.H. van der MPAFMDR. 2016. 
ESC Guidelines for the diagnosis and treatment of acute and chronic heart failure. Eur. Heart J. 37: 2129-200. DOI: 10.1002/ejhf.592.

18. Pugliese N.R., Masi S., Taddei, S. 2020. The renin-angiotensin-aldosterone system: a crossroad from arterial hypertension to heart failure. Heart Fail. Rev. 25, 31-42. doi.org/10.1007/s10741-01909855-5.

19. Radosinska J., Barancik M., Vrbjar N. 2017. Heart failure and role of circulating MMP-2 and MMP-9. Panminerva Med., 59(3): 241-253. DOI: 10.23736/S0031-0808.17.03321-3.

20. Sanchis L. 2015. Prognosis of new-onset heart failure outpatients and collagen biomarkers. Eur. J. Clin. Invest., 45: 842-849.

21. Simmonds S., Cuijpers I., Heymans S., Jones E. 2020. Cellular and Molecular Differences between HFpEF and HFrEF: A Step Ahead in an Improved Pathological Understanding. Cells, 9 (1): 242. https://doi.org/10.3390/cells9010242

22. Toba H, Lindsey M.L.. 2019. Extracellular matrix roles in cardiorenal fibrosis: Potential therapeutic targets for CVD and CKD in the elderly. Pharmacol. Ther., 193: 99-120. DOI: 10.1016/j.pharmthera.2018.08.014.

\section{References}

1. Vologdina I.V., Simanenkov V.I., Poroshina E.G., Min'ko B.A. 2016. Kachestvo zhizni i priverzhennost' terapii u pacientov pozhilogo i starcheskogo vozrasta s hronicheskoj serdechnoj nedostatochnost'ju, komorbidnymi kognitivnymi i affektivnymi narushenijami [Quality of life and adherence in patients with heart failure, cognitive and affective impairment]. Vestnik Severo-Zapadnogo gosudarstvennogo medicinskogo universiteta im. I.I. Mechnikova, 1 (8): 45-49.

2. Drapkina O.M., Drapkina Ju.S. 2012. Fibroz i aktivnost' renin-angiotenzin-al'dosteronovoj sistemy [Fibrosis and renin-angiotensin-aldosterone system activity]. Realii i perspektivy. Arterial'naja gipertenzija, 18 (5): 449-458.

3. Klinicheskie rekomendacii. Hronicheskaja serdechnaja nedostatochnost' (HSN). 2016 [Clinical guidelines. Chronic heart failure (CHF)]. Obshhestvo specialistov po serdechnoj nedostatochnosti i Rossijskoe kardiologicheskoe obshhestvo. $92 \mathrm{s.}$

4. Mareev V.Ju., Fomin I.V., Ageev F.T., Arutjunov G.P., Begrambekova Ju.L., Belenkov Ju.N., Vasjuk Ju.A., Galjavich A.S., Garganeeva A.A., Gendlin G.E., Giljarevskij S.R., Glezer M.G., Drapkina O.M., Dupljakov D.V., Kobalava Zh.D., Koziolova N.A., Lopatin Ju.M., Mareev Ju.V., Moiseev V.S., Nedoshivin A.O., Perepech N.B., Sitnikova M.Ju., Skibickij V.V., Tarlovskaja E.I., Chesnikova A.I., Shljahto E.V. 2017. Klinicheskie rekomendacii. Hronicheskaja serdechnaja nedostatochnost' (HSN) [Clinical guidelines. Chronic heart failure (CHF)]. Serdechnaja Nedostatochnost', 18 (3): 3-40. DOI: $10.18087 /$ rhfj.2017.1.2346.

5. Mjasoedova E.I. 2016. Soderzhanie matriksnoj metalloproteinazy-1 i ee ingibitora u pacientov $\mathrm{s}$ ishemicheskoj kardiomiopatiej [Content of matrix metalloproteinase-1 and its inhibitor in patients with ischemic cardiomyopathy]. Vestnik novyh medicinskih tehnologij, 23 (4): 50-53 DOI: 10.12737/23850.

6. Osipova O.A. Nagibina A.I., Komisov A.A., Petrova G.D., Shehovcova L.V., Vlasenko M.A., Vlasenko O.A. 2016. Patomorfologicheskie mehanizmy reguljacii obrazovanija miokardial'nogo fibroza u bol'nyh hronicheskoj serdechnoj nedostatochnost'ju na fone ishemicheskoj bolezni serdca [Pathomorphological mechanisms of the regulation of the formation of myocardial fibrosis in patients with chronic heart failure against ischemic heart disease]. Zhurnal serdechnaja nedostatochnost', 17 (5): 357-364. DOI: 10.18087/RHFJ.2016.5.2137.

7. Osipova O.A., Nikitina V.V., Shehovcova L.V., Nagibina A.I., Vlasenko M.A. 2015. Nejrogumoral'nye moduljatory $\mathrm{v}$ lechenii bol'nyh s hronicheskoj serdechnoj nedostatochnost'ju [Neurohumoral modulators in the treatment of patients with chronic heart failure]. Fundamental'nye issledovanija. 1 (8): 1655-1658.

8. Popov M.A., Shumakov D.V., Zybin D.I., Gurevich L.E., Ashevskaja V.E., Babokin V.E., Pronina V.P. 2019. Fenomen roli kollagena IV tipa i matriksnoj metalloproteinazy-9 tipa v remodelirovanii miokarda levogo zheludochka pri ishemicheskoj bolezni serdca [Role of type IV collagen and matrix metalloproteinase-9 in remodeling of the left ventricular in coronary artery disease]. Rossijskij kardiologicheskij zhurnal, 24 (8): 83-87. DOI: 10.15829/1560-4071-2019-8-83-87.

9. Reznik E.V., Nikitin I.G. 2019. Kardiorenal'nyj sindrom u bol'nyh s serdechnoj nedostatochnost'ju kak jetap kardiorenal'nogo kontinuuma (chast' I): opredelenie, klassifikacija, patogenez, diagnostika, jepidemiologija (obzor literatury) [Cardiorenal syndrome in patients with chronic heart failure as 
a stage of the cardiorenal continuum (part I): Definition, classification, pathogenesis, diagnosis, epidemiology]. Arhiv" vnutrennej mediciny, 9 (1): 5-22. DOI: 10.20514/2226-6704-2019-9-1-5-22.

10. Burke R.M., Lighthouse J.K., Mickelsen D.M., Small E.M. 2019. Sacubitril/Valsartan Decreases Cardiac Fibrosis in Left Ventricle Pressure Overload by Restoring PKG Signaling in Cardiac Fibroblasts. Circ. Heart Fail., 12 (4): e005565. DOI: 10.1161/CIRCHEARTFAILURE.118.005565.

11. Cleland J.G.F., Lyon A.R., Mcdonahh T., McMurray J.J.V. 2020. The year in cardiology: the year in cardiology 2019, European Heart Journal, 41 (12): 1232-1248, https://doi.org/10.1093/eurheartj/ehz949.

12. Ferreira J.M., Ferreira S.M., Ferreira M.J., Falcão-Pires I. 2017. Circulating Biomarkers of Collagen Metabolism and Prognosis of Heart Failure with Reduced or Mid-Range Ejection Fraction. Curr. Pharm. Des., 23 (22): 3217-3223. DOI: 10.2174/1381612823666170317124125.

13. Horn M.A, Trafford A.W. 2016. Aging and the cardiac collagen matrix: Novel mediators of fibrotic remodelling. Journal of Molecular and Cellular Cardiology 93, 175-185. DOI: 10.1016 / j. yjmcc.2015.11.005.

14. Kunkel G.H., Kunkel C.J., Ozuna H., Miralda I., Tyagi S.C. 2019. TFAM overexpression reduces pathological cardiac remodeling. Molecular and cellular biochemistry, 454 (1-2), 139-152. https://doi.org/10.1007/s1 1010-018-3459-9.

15. Morishita T., Uzui H., Mitsuke Ya., Amaya N., Kaseno K., Ishida K. 2017. Association between matrix metalloproteinase-9 and worsening heart failure events in patients with chronic heart failure. ESC Heart Failure; 4: 321-330. DOI: 10.1002/ehf2.12137.

16. Münzel T., Gori T., Keaney J.F.Jr., Maack C., Daiber A. 2015. Pathophysiological role of oxidative stress in systolic and diastolic heart failure and its therapeutic implications. European heart journal, 36 (38): 2555-2564. https://doi.org/10.1093/eurheartj/ehv305.

17. Ponikowski P., Voors A.A., Anker S.D., Bueno H., Cleland J.G., Coats A.J., Falk V., González-Juanatey J.R., Harjola V.P., Jankowska E.A., Jessup M., Linde C., Nihoyannopoulos P., Parissis J.T., Pieske B., Riley J.P., Rosano G.M., Ruilope L.M., Ruschitzka F., Rutten F.H. van der MPAFMDR. 2016. ESC Guidelines for the diagnosis and treatment of acute and chronic heart failure. Eur. Heart J. 37: 2129200. DOI: 10.1002/ejhf.592.

18. Pugliese N.R., Masi S., Taddei, S. 2020. The renin-angiotensin-aldosterone system: a crossroad from arterial hypertension to heart failure. Heart. Fail. Rev. 25, 31-42. doi.org/10.1007/s10741-01909855-5.

19. Radosinska J., Barancik M., Vrbjar N. 2017. Heart failure and role of circulating MMP-2 and MMP-9. Panminerva Med., 59 (3): 241-253. DOI: 10.23736/S0031-0808.17.03321-3.

20. Sanchis L. 2015. Prognosis of new-onset heart failure outpatients and collagen biomarkers. Eur. J. Clin. Invest., 45: 842-849.

21. Simmonds S., Cuijpers I., Heymans S., Jones E. 2020. Cellular and Molecular Differences between HFpEF and HFrEF: A Step Ahead in an Improved Pathological Understanding. Cells, 9 (1): 242. https://doi.org/10.3390/cells9010242.

22. Toba H., Lindsey M.L. 2019. Extracellular matrix roles in cardiorenal fibrosis: Potential therapeutic targets for CVD and CKD in the elderly. Pharmacol. Ther., 193: 99-120. doi:10.1016/j.pharmthera.2018.08.014.

\section{Ссылка для цитирования статьи Link for article citation}

Гостева Е.В., Шепель Р.Н., Осипова О.А. 2020. Взаимосвязь альдостерона и маркеров фиброза в патогенезе хронической сердечной недостаточности с промежуточной фракцией выброса в разных возрастных группах. Актуальные проблемы медицины, 43 (2): 216-228. DOI 10.18413/2687-0940-2020-43-2-216-228.

Gosteva E.V., Shepel R.N., Osipova O.A. 2020. Relationship of aldosterone and fibrosis markers in the pathogenesis of chronic heart failure by the middle range ejection fraction in different age groups. Challenges in Modern Medicine, 43 (2): 216-228 (in Russian). DOI 10.18413/2687-0940-2020-43-2-216-228. 